\title{
Strength and Conditioning Coaches' Application of the Session Rating of Perceived Exertion Method of Monitoring Within Professional Rugby Union
}

\author{
by \\ Thomas Comyns ${ }^{1,2}$, Aoife Hannon ${ }^{1}$
}

Session rating of perceived exertion (session-RPE) is a method of monitoring and managing training loads. The purpose of this study was to research how and for what purpose strength and conditioning (SEC) coaches implement this monitoring method within professional rugby union. The study also aimed to assess if SEC coaches found this monitoring method to be valid and effective. An online survey containing 24 fixed response questions was used to assess how SEC coaches applied the session-RPE method. The survey was piloted with expert researchers and practitioners in the area of session-RPE prior to distribution and alterations were made to the survey based on the experts' feedback. Twenty SEC coaches working with professional rugby union clubs in Ireland, England, Scotland and Wales completed the survey. The coaches' responses indicated that the majority found the session-RPE to be a valid and effective monitoring method. While some good implementation practices were identified, not all of the coaches adhered to these guidelines which may impact on the accuracy of the collected data. For example, 30\% of coaches do not collect the RPE for every session that a player does per week limiting the use of the session-RPE variables cumulative training load, training monotony, training strain and acute:chronic load ratio. SEC coaches within rugby should consider using session-RPE as a method of monitoring and implement the method in a manner reflective of research findings to enhance the potential applications of this system in maximising adaptations and minimising the risk of injury.

Key words: monitoring, training monotony, training load, training strain, acute:chronic load ratio.

\section{Introduction}

Rugby union is a high-intensity intermittent collision-based sport in which activities that rely on maximal strength, speed, and power are interspersed with periods of lower intensity aerobic activity and rest (Nicholas, 1997). It requires players to be proficient in rugbyrelated skills and to have a high fitness level in many areas, such as endurance, strength, power, speed and agility (Appleby et al., 2012). Successful training programs must involve overloading, but also must avoid the combination of excessive overloading plus inadequate recovery (Meeusen et al., 2012). Training volumes and intensities that are not optimal do not induce the desired physiological adaptations, whereas those that are excessive can potentially increase injury risk and impair sporting performance (Comyns and Flanagan, 2013). Monitoring and quantifying the training stimuli that a rugby player is exposed to are necessary to prevent the negative consequences associated with excessive training and are central to performance (Comyns and Flanagan, 2013). Such monitoring can assist with load management, which aims to optimally

1 - Department of Physcial Education and Sport Sciences, University of Limerick, Limerick, Ireland.

${ }^{2}-$ Health Research Institute, University of Limerick, Limerick, Ireland. 
configure training to maximise adaptation with minimal injury risk (Soligard et al., 2016).

Numerous techniques and methods are available to $S \& C$ coaches working within rugby to quantify the training stimuli in order to optimise the training adaptations and minimise the incidence of illness and injury. Excessive training loads have been identified as a risk factor for injury in various sports, such as rugby (Brooks et al., 2008) and rugby league (Gabbett and Jenkins, 2011). Session-RPE is utilised within rugby union as a method of monitoring training and providing coaches with subjective information of the internal load experienced by players across all rugby training modalities (Comyns and Flanagan, 2013). Subjective measures of internal loads are more sensitive and consistent than objective measures in determining acute and chronic changes in a player's well-being in response to loads (Saw et al., 2016). Session-RPE is a simple, inexpensive and non-invasive monitoring method based on the Borg's category ratio 10 (CR-10) scale (Borg, 1982). The duration of each session is recorded and this value is multiplied by the player's global rating of the intensity of the session using the CR-10 to give a score for a training load. A number of variables can be derived from this training load data, namely weekly training load, training monotony and training strain. Weekly training load is calculated by summating all of the session training load scores for that week. The daily training load (summation of all session training load values for one day) scores are used to derive a score for training monotony, which is a measure of the dayto-day training variability during a training week (Foster, 1998). Training monotony is calculated by dividing the mean daily training load by the standard deviation of the daily training load over a 1-week period (Comyns and Flanagan, 2013). In addition, the weekly training load score is multiplied by the training monotony score to calculate training strain, which is a value that represents the overall stress that the player was exposed to throughout the training week (Foster, 1998).

According to Quarrie et al. (2016), measuring load at professional rugby union level should incorporate the session-RPE method. This method is considered to be a simple and easily implemented system within rugby union that provides an effective means of monitoring a player's response to load (Quarrie et al., 2016). Research has shown that the session-RPE is a reliable tool to assess training load in steady state aerobic training (Foster et al., 2011), intermittent aerobic training (Foster et al., 2011) and strength training (Day et al., 2014). Clarke et al. (2013) researched the validity of the session-RPE method for collision-based field sports and reported strong significant correlations between sessionRPE and heart rate training impulse $(r=0.65-0.91$ observed between session-RPE and Polar TRIMP during soccer practice sessions). Session-RPE is highly practical and accurately measures an individual's internal training load within collision based sports (Clarke et al., 2013). It can be used to monitor all forms of training that are involved in the physical preparation of professional rugby union players and thus provides a global measure of the players' entire weekly training load. In addition, session-RPE derived variables have the potential to identify a player's increased risk of illness and injury (Foster, 1998; Gabbett, 2010; Gabbett and Jenkins, 2011) illustrating the application of this monitoring method in injury and illness prevention and management. However, there are session-RPE implementation guidelines that need to be followed when using the session-RPE in order to enhance the accuracy and potential applications of the collected data. Research indicates, for example, that the players should provide their rating of the global intensity of the session either 10 minutes (Uchida et al., 2014), 15 minutes (Kraft et al., 2014) or 30 minutes (Clarke et al., 2013; Foster et al., 2011) post cessation of the session so that the rating reflects the global intensity of the session. Christen et al. (2016), however, reported that post-exercise time does not appear to have a significant effect on session-RPE after either steady-state or interval exercise and that session-RPE can be reported 5 minutes after the end of the training session. It is unknown if such a finding would be related to a collision based sport and more research is needed in such a sporting context on post-exercise session-RPE timing. In addition, ratings of session-RPE need to be recorded for all training sessions that the players are exposed to per week in order to accurately calculate overall training load (daily and weekly), training monotony and training strain. 
While there is extensive research examining the validity of the session-RPE within team sports (Clarke et al., 2013; Impellizzeri et al., 2004; Scott et al., 2013), limited research exists on how the session-RPE is applied within this setting. Only one applied review article by Comyns and Flanagan (2013) has outlined how the session-RPE can be utilised within professional rugby union. The authors noted that the session-RPE provides subjective, valid and reliable information about the internal training load experienced by rugby players across all modalities of training. The system can be used to provide information on within-week and acrossweek training load within professional rugby (Comyns and Flanagan, 2013). Session-RPE can assist with squad training load management, provided only 'fit to play' players are included in squad averages, and individual player training load management, in particular players returning from acute and chronic injury (Comyns and Flanagan, 2013). Consequently, Comyns and Flanagan (2013) highlighted the potential use of the session-RPE method for tracking periodised training blocks and tapering phases of training. In addition, the authors noted the contribution of training monotony and training strain data to monitoring within professional rugby union. Both these variables can identify weeks where training load has not been appropriately managed and arranged, allowing coaches subsequently organise training in a more optimal manner (Comyns and Flanagan, 2013).

The session-RPE method has clear applications and advantages as a method of monitoring and managing player load in professional rugby union in order to maximise adaptations and performance and minimise the risk of injury and illness. Certain implementation guidelines should be adhered to optimise the potential use of this monitoring method. No research has previously been undertaken to assess how the session-RPE method is used by S\&C coaches within professional rugby union. It is unknown if these coaches find the method effective and valid for use with rugby squads, individual players and injured players. Therefore, the purpose of this study was to establish if S\&C coaches find the session-RPE method valid and effective and to assess how and for what purpose the coaches implemented the session-RPE within professional rugby union.

\section{Methods}

\section{Participants}

Twenty S\&C coaches who worked with different professional rugby clubs in Ireland, England, Wales and Scotland completed the online survey. All of the coaches worked for clubs that took part in European professional rugby competitions. In addition, the coach had to use the session-RPE within the club as a method of monitoring training. Only one contacted coach did not use the session-RPE method and they were thus excluded from participating. The coaches had on average $11.2 \pm 5.9$ years' experience working in the area of strength and conditioning. Approval for the use of human subjects was obtained from the University of Limerick ethical review board. All subjects were informed that participation in the study was voluntary, that responses were anonymous, and that they could withdraw from participation at any stage.

Procedures

This study involved the use of an online survey (SurveyMonkey, Palo Alto, CA, USA) to assess the application of the session-RPE method of monitoring training in professional rugby union. An introductory letter describing the project and the survey was emailed to the lead $S \& C$ coach working with professional teams in Ireland, England, Scotland and Wales. The purpose of the letter was to explain the survey, the time commitment and the confidentiality of all collected information. Coaches were also informed that they could exit the survey at any stage without any implication. Twenty coaches volunteered to participate in the study and they were subsequently sent a SurveyMonkey (SurveyMonkey, Palo Alto, CA, USA) link to the online version of the finalised survey after the signing of an informed consent document. The coaches were given 30 days from the time they received the SurveyMonkey link to complete the survey. If no response was received after two weeks a reminder email containing the survey link was sent to the coach. After 30 days, all completed responses were downloaded from the SurveyMonkey site and collated for statistical analysis. 


\section{Survey}

The survey was initially designed based on the literature related to the use of the session-RPE, particularly within rugby union (Comyns and Flanagan, 2013; Quarrie et al., 2016). This initial survey was piloted with a panel of eight expert practitioners and researchers with a renowned expertise in implementing and researching the session-RPE method of monitoring training. The expert panel group were asked to provide feedback on whether or not the survey matched the purpose of the study, on the content of the questions and the structure of the survey. Feedback from the expert panel was positive with one panel member commenting that the survey was 'an excellent survey and very comprehensive'. The panel did provide some specific recommendations with regard to survey structure and the wording of questions. As a result, slight modifications were made to the structure of the survey and the terminology used within a small number of questions. The final amended survey consisted of 24 fixed response questions related to three main areas:

1. Coaches' implementation of the session-RPE method. The questions in this section sought to gather data on how the coaches implemented the method in the practical setting. It included, for example, questions on when the RPE was reported for each session by the players, how the RPE was collected from the players, whether or not the RPE was collected for all sessions, and if training load, training monotony and training strain were calculated from the raw data.

2. Coaches' application of the data collected through the session-RPE method. This section contained questions related to the applications that the coaches used the data and the derived variables for. The coaches were asked if they used the session-RPE method to guide and assess their periodised program, to assess individual and team response to a taper, and to monitor squad averages and injured players (acute and chronic).

3. Coaches opinion of whether the session-RPE is valid and if it is an effective method of monitoring training. In particular, the survey contained questions that asked the coaches if they thought the session-RPE method was effective in monitoring individual players, squad averages and injured players (acute and chronic). In addition, the coaches were asked if they found the session-RPE method effective in preventing injury, preventing illness and enhancing performance.

\section{Statistical Analyses}

As the survey contained fixed response questions, all of the subject responses were analysed descriptively using Microsoft Excel. The descriptive statistics involved calculating and reporting percentages, and frequencies of responses.

\section{Results}

The results of the survey are presented as frequencies of responses together with percentages and are divided up into three sections in line with the structure of the survey. Each section details the responses for questions related to the session-RPE method. Additionally, the participants were asked if they relied solely on the session-RPE to quantify the training stimuli that their rugby players were exposed to. Only one participant $(5 \%)$ relied solely on this method of monitoring.

\section{Coaches' implementation of the session-RPE method}

This section details the results related to how the coaches implemented the session-RPE within their respective clubs. In Table 1 the coaches' responses are presented on whether or not they used the session-RPE method for all sessions that the players were exposed to. The coaches' responses on whether or not they calculated the session-RPE variables of training load, monotony and strain are also detailed in Table 1. The session-RPE method is commonly used to provide coaches with an overview of the workload for a squad of players across varying training modalities (Comyns and Flanagan, 2013). Central to this is the calculation of the squad average for the variables derived via the sessionRPE method. The participants were asked to indicate which categories of players they used in the calculation of their squad average data and the results are presented in Table 1.

The coaches were asked at what time point they collected the players' RPE post cessation of a training session or a game. Fortyone percentage $(n=7)$ indicated that they did this 
immediately, $53 \%(n=9)$ collected this data after 15 minutes and $6 \%(n=1)$ collected this data after 30 minutes. The coaches were also asked what method they used to collect the session-RPE from the players and $89 \%(n=17)$ indicated that this was done verbally, while $11 \%(n=2)$ used a mobile application to collect this data. No coaches used text messages to collect the session-RPE post cessation of a session or game. The $S \& C$ coaches were asked if they educated the players and rugby coaches about the session-RPE before implementing it as a monitoring tool and $85 \%(\mathrm{n}=$ 17) said they educated both players and coaches. The remaining $15 \%(\mathrm{n}=3)$ only educated the players on the session-RPE method prior to its use within the club.

\section{Coaches' application of the data collected through the session-RPE method}

In Table 2 some of the results related to what the coaches used the session-RPE method for are presented. This table provides detail on the coaches' use of the session-RPE with injured (acute and chronic) players and their use of this method to monitor the periodisation of training programs and the players' response to a taper period of such a program. The coaches were asked to indicate if they used the session-RPE method to calculate training load, training monotony and training strain in order to monitor individual players and the rugby squad average. These results are presented in Figure 1.

Coaches opinion of whether the session-RPE is valid and if it is an effective method of monitoring training

Sixteen of the coaches $(80 \%)$ responded that they found the session-RPE to be a valid method to quantify training stimuli. The coaches were also asked if they thought the session-RPE method was effective in monitoring various player categories within rugby union. The relevant results are presented in Figure 2. In addition, the coaches' viewpoint on whether or not they found the monitoring method effective in preventing injury, preventing illness and enhancing performance is detailed in Figure 3.

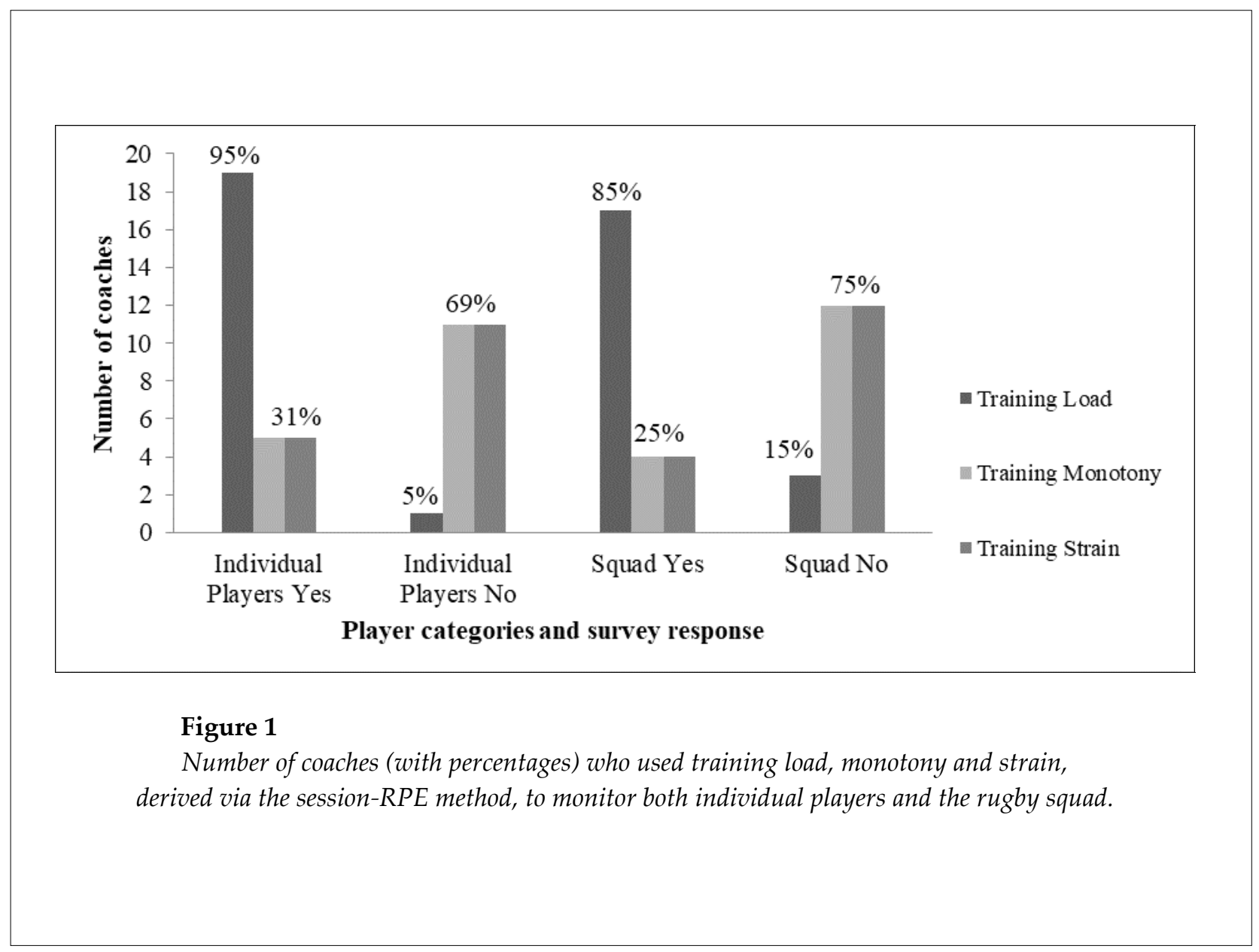

(C) Editorial Committee of Journal of Human Kinetics 

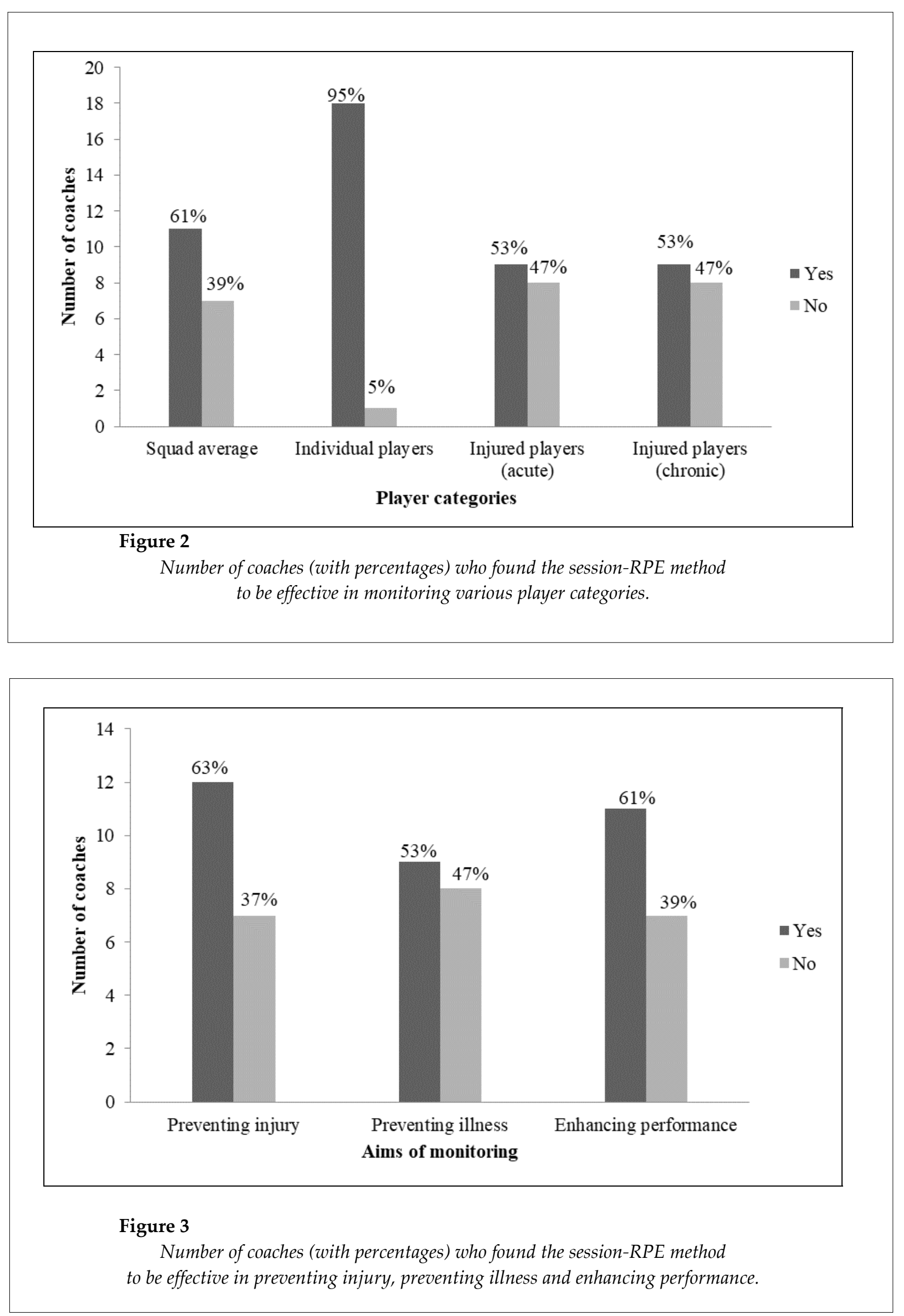
Table 1

Results related to the SEC coaches' responses to questions regarding their implementation of the session-RPE method

\begin{tabular}{lll}
\hline $\begin{array}{l}\text { Session-RPE Implementation Questions } \\
\text { Do you... }\end{array}$ & $\begin{array}{l}\text { Yes } \\
\mathrm{n}(\%)\end{array}$ & $\begin{array}{l}\text { No } \\
\mathrm{n}(\%)\end{array}$ \\
\hline $\begin{array}{l}\text { 1. Collect session-RPE for all aspects of training that the players are exposed to } \\
\text { 2. Use session-RPE method to calculate Training Load }\end{array}$ & $\begin{array}{l}14(70 \%) \\
20(100 \%)\end{array}$ & $\begin{array}{l}6(30 \%) \\
0(0 \%)\end{array}$ \\
$\begin{array}{l}\text { 3. Use session-RPE method to calculate Training Monotony } \\
\text { 4. Use session-RPE method to calculate Training Strain }\end{array}$ & $6(32 \%)$ & $13(68 \%)$ \\
& $7(39 \%)$ & $11(61 \%)$ \\
$\begin{array}{l}\text { 5. Use the following player categories in the calculation of your squad average } \\
\text { data: }\end{array}$ & & \\
$\begin{array}{l}\text { Fully-fit players } \\
\text { Players who complete the majority of the session } \\
\text { Players on a rehab program }\end{array}$ & $18(100 \%)$ & $0(0 \%)$ \\
& $13(76 \%)$ & $13(76 \%)$ \\
\end{tabular}

Table 2

Results related to the $S \mathcal{E} C$ coaches' responses to questions regarding their application of the data collected via the session RPE method

\begin{tabular}{|c|c|c|}
\hline $\begin{array}{l}\text { Session-RPE Application Questions } \\
\text { Do you... }\end{array}$ & $\begin{array}{l}\text { Yes } \\
\mathrm{n}(\%)\end{array}$ & $\begin{array}{l}\text { No } \\
\mathrm{n}(\%)\end{array}$ \\
\hline 1. Use the session-RPE method to guide and assess your periodised training program & $10(50 \%)$ & $10(50 \%)$ \\
\hline 2. Use the session-RPE method to assess response to a taper & $10(50 \%)$ & $10(50 \%)$ \\
\hline $\begin{array}{l}\text { 3. Use the session-RPE method to monitor players with acute injury in their return to } \\
\text { full training }\end{array}$ & $13(65 \%)$ & $7(35 \%)$ \\
\hline $\begin{array}{l}\text { 4. Use the session-RPE method to monitor players with chronic injury in their return } \\
\text { to full training }\end{array}$ & $13(68 \%)$ & $6(32 \%)$ \\
\hline
\end{tabular}

\section{Discussion}

The results of the survey provide insight into how the session-RPE is implemented within professional rugby union, the applications that the $S \& C$ coaches use the collected data for and whether or not they find the method valid and effective. The majority of coaches surveyed reported that the session-RPE was a valid and effective method of monitoring. Half of the coaches used the session-RPE method to guide and assess periodised training programs and the players' response to a taper phase. It is evident that some good implementation practices were undertaken by the coaches, such as player and coach education related to session-RPE. However, there were certain implementation guidelines that were not adhered to by all the coaches which may have an impact on the accuracy and effectiveness of the collected data. Such implementation practices can potentially limit the number of applications that the $S \& C$ coaches can use the session-RPE method for.

Comyns and Flanagan (2013) outlined 
session-RPE implementation guidelines with regard to professional rugby union. Potential errors can arise with any data collection system and consistency of data collection and adherence to implementation guidelines are essential to ensure accuracy. Research has indicated that players should provide their RPE for any session either 10 minutes (Uchida et al., 2014), 15 minutes (Kraft et al., 2014) or 30 minutes (Clarke et al., 2013; Foster et al., 2011) post cessation of the session. While the majority of the S\&C coaches in this study used either the 15 or 30 minute time point to collect the session-RPE, $41 \%$ took the RPE immediately after the session finished. Such practice is contrary to the implementation guidelines (Comyns and Flanagan, 2013; Turner and Bishop, 2015) and research findings (Clarke et al., 2013; Foster et al., 2011; Kraft et al., 2014; Uchida et al., 2014) and may have a potential impact on the accuracy of the collected data for this cohort of coaches. However, it is not contrary to research by Christen et al. (2016) who indicated that post-exercise time did not have an effect on session-RPE. It is worth noting though that the research by Christen et al. (2016) was not conducted on a collision based sport and thus these findings regarding the temporal robustness of the session-RPE may not be applicable in the current context. In addition, $89 \%$ of coaches collected the session-RPE verbally from the players as opposed to using a mobile phone application or text response. While collecting ratings verbally from players may be more practical from a logistically viewpoint, it does pose some issues with regard to conscious bias. Conscious bias is often the result of an individual reporting in a socially desirable manner, generally over-reporting favourable responses and underreporting unfavourable ones (Saw et al., 2015). Potentially in a competitive team setting, players may under-report a RPE for a session verbally in front of peers ('faking good') to appear to be coping with training in order to gain selection ahead of a teammate competing for the same position (Ekegren et al., 2014). Players who report RPE verbally may also be influenced by the RPE reported by other players. Thus reporting the session-RPE in private via a mobile phone application, for example, may increase the accuracy of the results and reduce the influence of conscious bias to a degree.
Session-RPE implementation guidelines indicate that the session-RPE should be reported for every session that the player undertakes per week in order to obtain a value for weekly training load and subsequently training monotony and training strain (Comyns and Flanagan, 2013; Turner and Bishop, 2015). Without an RPE and session duration for every session that a player takes part in, daily and weekly training load, training monotony and training strain cannot be calculated. Recently cumulative training load has been used to calculate the acute:chronic load ratio, which describes the acute training load (training load for the last week) to the chronic load (the 4-week rolling average load) relationship (Gabbett, 2016). This emerging concept has been validated in a number of sports, namely Australian football, cricket and rugby league (Blanch and Gabbett, 2016). Research by Williams et al. (2017) identified a systematic process of data reduction and variable selection when using the session-RPE method within rugby union. The results indicated that for elite collision sports, such as rugby union, a measure of cumulative load (4-week cumulative load), change in load (acute:chronic load) and acute load (daily training load) should be monitored for injury risk management purposes. While the majority of $S \& C$ coaches surveyed in the current study collected the RPE for every session that the players were exposed to, $30 \%$ did not follow this protocol. These coaches were unable to derive the variables of training load (cumulative and daily), monotony and strain due to the fact that not every session was included in their session-RPE monitoring. Such practice places substantial limitations on the use of the session-RPE for these coaches and does not allow for the recording of the essential session-RPE variables for rugby union as identified by Williams et al. (2017). It is also impossible for these coaches to calculate acute:chronic load ratios for their players which provide some clear information on how the load is periodised across a cycle. The use of the acute:chronic load ratio identifies when there is an excessive and rapid increase in load which has been associated with increasing injury risk (Gabbett et al., 2016). Gabbett (2016) indicated that there was a reduced risk of injury if the acute:chronic load ratio was within the range from 0.8 to 1.3 and that injury 
risk increased when this ratio exceeded 1.5. It is worth noting that these ranges and values are guidelines and there may be differences based on the sport. For $30 \%$ of coaches who did not collect session-RPE data for every session, they did not utilise the method to its potential and thus limited its use as a method to identify players who were at risk of injury.

Training monotony and training strain provide the coach, respectively, with information related to the variability of the prescribed training and the overall training stress that the player was exposed to (Comyns and Flanagan, 2013). These variables can assist with assessing the player's response to training to ensure that the training stimuli are periodised in an appropriate manner. The majority of coaches responded that they did not calculate training monotony $(68 \%)$ and training strain (61\%). Similarly, the majority of coaches did not use either variable to monitor individual players or squad average data. These coaches did not utilise the full potential of the session-RPE method and limited the effectiveness of this method to assist in the prevention of illness and injury. Training plans that do not alter between hard and easy training days can inflate training monotony and training strain scores and are associated with incidences of illness and overtraining (Foster et al., 1998).

Only half of the coaches responded that they used the session-RPE method to guide and assess both their periodised program and the players' response to a taper period within this program. A central application and advantage of the session-RPE method is the assessment and monitoring of both periodised programs and the impact of planned taper weeks (Comyns and Flanagan, 2013; Turner and Bishop, 2015). Coutts et al. (2009) used the session-RPE variables of training load, monotony and strain to describe the periodisation of professional rugby league training across a full training year. Specifically, Comyns and Flanagan (2013) indicated that for professional rugby union the session-RPE method can provide squad average information related to training load, monotony and strain on acrossweek training loading. Such information allows the coach to assess where heavy and light weeks have occurred and whether or not the players' perception of training is consistent with the periodised plan (Comyns and Flanagan, 2013).
Half of the coaches surveyed in this study did not use the session-RPE method for this purpose and thus, limited the impact that this monitoring method can have on squad load management within professional rugby union. Regarding the squad load management, Comyns and Flanagan (2013) indicated that only 'fit to play' players should beincluded in any squad average calculations. While all of the coaches included fully fit players in their squad average calculations, $76 \%$ included players who completed the majority of the session and $21 \%$ included players on a rehab program. Such practices impact on the accuracy of squad average calculations which in turn can cause inconsistencies with data reporting and interpretation.

While the session-RPE method has applications for load monitoring and management with individual players and a squad within professional rugby union, it can also be utilised to manage players with either acute or chronic injuries (Comyns and Flanagan, 2013). The method can be used to monitor adapted training plans and develop these plans so that they 'mirror' full team training (Comyns and Flanagan, 2013). This is another potential application of the session-RPE method. The responses to the survey indicate that $35 \%$ of coaches did not use it to monitor players with acute injury and $32 \%$ did not utilise the method with chronically injured players. These coaches again limited the application of this versatile method to assist with the management of injured players.

All but one coach responded that they found the session-RPE method effective in the monitoring of individual players. The majority of coaches responded that they thought the method was effective in monitoring the squad and injured players, although this percentage was $53 \%$ for the latter. In addition, while the majority of the coaches responded that they found the method effective in preventing illness and injury and enhancing performance, the reported percentages ranged from 53 to $63 \%$. Research has reported that excessive and rapid increases in load are associated with increased injury risk (Foster et al., 1998; Gabbett, 2016). Quarrie et al. (2016) reported that the session-RPE method was an effective means of monitoring a rugby union player's 
response to load. There are numerous applications of the session-RPE that make it an effective monitoring method, such as the monitoring of individual players, injured players, and the management of periodised programs and taper cycles to minimise injury risk and maximise adaptations. As is evident from the results of this survey, there are implementation issues regarding the use of this monitoring method within professional rugby union which may limit the application of the method and the accuracy of the collected data. Such restrictions may have had an influence on some of the coaches' viewpoint regarding the effectiveness of the session-RPE method. It would have been beneficial to have conducted a semi-structured interview with these coaches to gain further insight into why they may not have found it effective. This is a limitation of the current study and further research should aim to use a semi-structured interview design to explore participants' experiences, opinions and to elicit truly open ended responses (Patton, 2015).

\section{Conclusion}

The responses to the survey in this study provided insight into how and why S\&C coaches used the session-RPE monitoring method within professional rugby union. The majority of coaches responded that they found the method to be effective in monitoring various player categories and preventing illness, injury and enhancing performance. There is evidence of good practice within rugby union regarding the application of the session-RPE method to minimise injury risk and maximise adaptations. The coaches in general undertake education with players and coaches prior to the implementation of the system and they use the session-RPE variable of training load to monitor individual players and the squad within the club. The findings of the survey highlighted that certain implementation guidelines were not fully adhered to when using this monitoring method. All coaches did not collect the player RPE either 15 or 30 minutes post session cessation and this RPE was not reported by all coaches for every session that the players undertook. This has an implication for these coaches on what variables can be calculated from the session-RPE monitoring which can restrict the use of the variables of cumulative training load, training monotony, training strain and acute:chronic load ratio.

From a practical viewpoint, coaches should consider the findings of research regarding these implementation guidelines prior to using the system. The results indicate that within professional rugby union $\mathrm{S} \& \mathrm{C}$ coaches find the method to be valid and effective. Coaches working with this population group should consider implementing the session-RPE method as a means of monitoring and managing load. Correct implementation of the session-RPE guidelines by $S \& C$ coaches, however, is important and would impact on the accuracy of the collected data and enhance the potential applications of these data sets to maximise adaptations and thus performance with minimal risk of injury.

\section{References}

Appleby B, Newton RU, Cormie P. Changes in strength over a 2-year period in professional rugby union players. J Strength Cond Res, 2012; 26: 2538-2546

Blanch, P, Gabbett TJ. Has the athlete trained enough to return to play safely? The

acute:chronic workload ratio permits clinicians to quantify a player's risk of

subsequent injury. Br J Sports Med, 2016; 50: 471-475

Borg G. Psychophysical basis of perceived exertion. Med Sci Sports Exerc, 1982; 14: 363-367

Brooks JH, Fuller CW, Kemp SP, Reddin BM. An assessment of training volume in professional rugby union and its impact on the incidence, severity, and nature of match and training injuries. J Sports Sci, 2008; 26: 863-873

Christen J, Foster C, Porcari JP, Mikat RP. Temporal robustness of the session rating of perceived exertion. Int J Sports Physiol Perf, 2016; 11(8): 1088-1093

Clarke N, Farthing, J, Norris S, Arnold B, Lanovaz J. Quantification of training load in Canadian football: application of session-RPE in collision-based team sports. J Strength Cond Res, 2013; 27(8): 2198-2205 
Comyns T, Flanagan EP. Applications of the session rating of perceived exertion system in professional rugby union. Strength Cond J, 2013; 35(6): 78-85

Coutts A, Sirotic A, Catterick C, Knowels H. Monitoring training loads in professional rugby league. In: Reilly T and Korkusuz F. Science and Football VI: Proceedings of the Sixth World Congress on Science and Football. London: Routledge, 272-277; 2009

Day M, McGuigan MR, Brice G, Foster C. Monitoring exercise intensity during resistance training using the session-RPE scale. J Strength Cond Res, 2004; 18: 353-358

Ekegren CL, Donaldson A, Gabbe BJ, Finch CF. Implementing injury surveillance systems alongside injury prevention programs: evaluation of an online surveillance system in a community setting. Injury Epidemiology, 2014; 1: 19

Foster C. Monitoring training in athletes with reference to overtraining syndrome. Med Sci Sports Exerc, 1998; 30(7): 1164-8

Foster C, Florhaug JA, Franklin J, Gottschall L, Hrovatin L, Parker S, Doleshal P, Dodge C. A new approach to monitoring exercise training. J Strength Cond Res, 2001; 15: 109-115

Gabbett TJ. The development and application of an injury prediction model for

noncontact, soft-tissue injuries in elite collision sport athletes. J Strength Cond Res, 2010;

24: $2593-2603$

Gabbett TJ. The training-injury prevention paradox: should athletes be training

smarter and harder? Br J Sports Med, 2016; 50: 273-280

Gabbett TJ, Jenkins DJ. Relationship between training load and injury in professional rugby league players. $J$ Sci Med Sport, 2011; 14(3): 204-209

Impellizzeri FM, Rampinini E, Coutts AJ, Sassi A, Marcora SM. Use of RPE-based training load in soccer. Med Sci Sports Exerc, 2004; 36: 1042-1047

Kraft JA, Green JM, Thompson KR. Session rating of perceived exertion responses during resistance training bouts equated for total work but differing in work rate. J Strength Cond, 2014; 28(2): 540-545

Meeusen R, Duclos M, Foster C, Fry A, Gleeson M, Nieman D, Raglin J, Rietjens G, Steinacker J, Urhausen A. Prevention, diagnosis, and treatment of the overtraining syndrome: joint consensus statement of the European College of Sport Science and the American College of Sports Medicine. Med. Sci. Sports Exerc, 2013; 45(1): 186-205

Nicholas CW. Anthropometric and physiological characteristics of rugby union football players. Sports Med, 1997; 23: 375-396

Patton MQ. Qualitative research and evaluation methods: integrating theory and practice. 4th ed. USA: SAGE Publications Inc; 2015

Quarrie KL, Raftery M, Blackie J, Cook CJ, Fuller CW, Gabbett TJ, Gray AJ, Gill N, Hennessy L, Kemp S, Lambert M, Nichol R, Mellalieu SD, Piscione J, Stadelmann J, Tucker R. Managing player load in professional rugby union: a review of current knowledge and practices. Br J of Sports Med, Epub ahead of print. DOI:10.1136/bjsports-2016-096191

Saw AE, Main, LC, Gastin P. Monitoring athletes through self-report: factors influencing implementation. J Sports Sci Med, 2015; 14: 137-146

Saw A, Main LC, Gastin P. Monitoring the athlete training response: subjective self-reported measures trump commonly used objective measures: a systematic review. Br J Sports Med, 2016; 50: 281-291

Scott TJ, Black CR, Coutts AJ. Validity and reliability of the session-RPE method for quantifying training in Australian football: a comparison of the CR10 and CR100 scales. J Strength Cond Res, 2013; 27(1): 270276

Soligard T, Schwellnus M, Alonso J-M, Bahr R, Clarsen B, Dijkstra HP, Gabbett T, Glesson M, Hägglund M, Hutchinson MR, van Rensburg CJ, Khan KM, Meeusen R, Orchard JW, Pluim BM, Raftery M, Budgett $R$, Engebretsen L. How much is too much? (Part 1) International Olympic committee consensus statement on load in sport and risk of injury. Br J Sports Med, 2016; 50: 1030-1041

Turner AN, Bishop C, Marshall G, Read P. How to monitor training load and mode using sRPE. Prof Strength Cond, 2015; 39: 15-20 
Uchida MC, Teixeira LFM, Godoi VJ, Marchetti PH, Conte M, Coutts AJ, Bacurau RFP. Does the timing of measurement alter session-RPE in boxers? J Sports Sci Med, 2014; 13: 59-65

Williams S, Trewartha G, Cross MJ, Kemp SPT, Stokes KA. Monitoring what matters: A systematic process for selecting training load measures. Int J Sports Physiol Perf, 2017; 12: S2-101-S2-106

\section{Corresponding author:}

\section{Thomas Comyns}

Department of Physical Education and Sport Sciences

University of Limerick

Limerick, Ireland

Phone: +35361234738

E-mail: tom.comyns@ul.ie 